\title{
Free Vibrations of Nanoscale Beam Under Two-Temperature Green and Naghdi model
}

\author{
Ibrahim A. Abbas \\ Department of Mathematics, Faculty of Science, Sohag University, Sohag, Egypt. \\ Nonlinear Analysis and Applied Mathematics Research Group (NAAM), Department of Mathematics, \\ King Abdulaziz University, Jeddah, Saudi Arabia.
}

(Received 9 March 2015; accepted 13 January 2016)

The influence of the coupling between strain rate and temperature becomes domineering in the nanoscale beam. In this work, the free vibration of nanoscale beam resonators is analysed using Green and Naghdis theorem under the two-temperature model (2TGNIII). The influence of two-temperature parameters in a nanoscale beam is studied for beams under simply supported conditions. Exact expressions for frequency shift and the thermoelastic damping have been derived in the resonator, and calculation outcomes have been presented graphically with respect to frequency shift, natural frequency, and thermoelastic damping. The scale of length and thickness for a nanobeam equal to $15 \times 10^{-9} \mathrm{~m}$ and equal to $1.3 \times 10^{-12} \mathrm{~s}$ for time.

\section{INTRODUCTION}

Many attempts have been made to study the elastic properties of nanostructured materials by atomistic simulations. Diao et al. ${ }^{1}$ investigated the influence of free surface on the body and elastic properties of gold nanowires using atomic emulations. Modelling and emulation of thermoelastic damping is a subject of repeated attention in the nanomechanics community and nanoengineering community, one most encouraged by nanoelectromechanical advancement system (NEMS) technologies. The systems of Nanoelectromechanical, or NEMS, reach quite high essential frequencies of procedure, especially when one considers their miniature size and small force constants. Such mechanical devices of high frequencies have many significant applications, among which are scanning probe microscopes, mechanical signal processing, and ultrasensitive mass detection.

Lord and Shulman ${ }^{2}$ extended the couple thermoelastic theory. Green and Lindsay ${ }^{3}$ included the thermal relaxation times in constitutive equations. The counterparts of our problems in the context of theories of thermoelastic theory were considered by using numerical and analytical approaches. ${ }^{4-9}$ Green and Naghdi ${ }^{10,11}$ established GNII and GNIII generalized thermoelastic models, which based the replacing of usual entropy inequality alongside the entropy equality. In recent years, various problems have been taken into account using the Green and Naghdi models. ${ }^{12-26}$

Thermoelasticity using two-temperature modelling is one of the unconventional thermoelastic models of elastic solids. Thermal dependence is the main variance from this theorem compared to classical theory. Chen et al. ${ }^{27-29}$ established a theorem of thermal conduction in deformable bodies, which based on two featured temperatures: thermodynamic and conductive. The variance between these two temperatures is proportionate to the thermal supply for time independent cases. For timedependent problems and problems of waves propagation in particular, the two temperatures are mostly different in cases of the presence and absence of the thermal input. Youssef ${ }^{30}$ presented the generalized thermoelastic theory under twotemperature by using Fourier law to the field equations. ElKaramany and Ezzat ${ }^{31}$ introduced the two-temperature GreenNaghdi thermoelasticity models. Abbas et al. ${ }^{32-34}$ presented various problems based on the two-temperature thermoelastic model using numerical and analytical methods.

Due to their numerous significant technological applica-

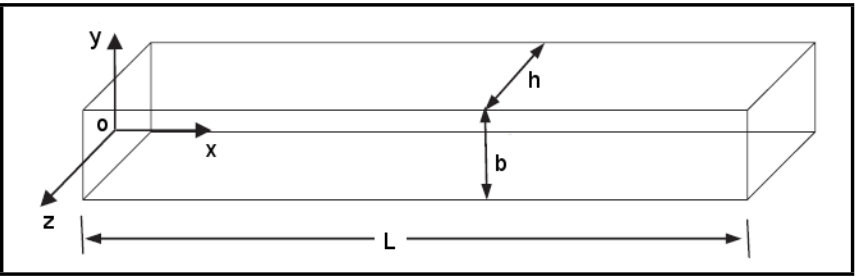

Figure 1. Schematic illustration of the beam setup.

tions, nanomechanical resonators have attracted considerable attention. The processes of vibration and heat transfer have been studied by many researchers. Honsten et al. ${ }^{35}$ predicted that the inner friction in 50-nm scale silicon-based MEMS structures is strong when subjected to thermoelastic damping. Nayfeh and Younis ${ }^{36-39}$ presented analytical expressions for the quality factor of microplates of general shapes subjected to thermoelastic damping. Rezazadeh et $\mathrm{al}^{40}$ used the modified couple stress model to study the thermoelastic damping in a microbeam resonator. Sun et al. ${ }^{41}$ investigated thermoelastic damping in microbeam resonators. Elsibai and Youssef ${ }^{42}$ applied the state-space method to study the vibration of the gold nanobeam due to ramp-type heating under Green and Naghdis type II model. Sharma ${ }^{43}$ investigated the frequency shift and thermoelastic damping in both microscale and nanoscale anisotropic resonators. Grover and Sharma ${ }^{44,45}$ studied the effect of relaxation time for thermoelastic vibration on MEMS and NEMS with voids.

This paper investigates the free vibrations of a nanobeam resonator in the context of the Green and Naghdi theory with two-temperature (2TGNIII). The impact of two-temperature parameters in the natural frequency, frequency shift, and the thermoelastic damping have been studied and are graphically represented.

\section{FORMULATIONS OF THE PROBLEM}

We consider the theoretical analysis of small flexural deflection of an isotropic, homogenous heat conductor, thermoelastic resonator by using the Cartesian coordinate system oxyz for the temperature increment $T(x, y, z, t)$ and the vector of displacement $u(x, y, z, t)=(u, v, w)$, which have the dimension thickness $h\left(-\frac{h}{2} \leq z \leq \frac{h}{2}\right)$, the width $b\left(-\frac{b}{2} \leq y \leq \frac{b}{2}\right)$, and the length $L(0 \leq x \leq L)$, as in Fig. 1. In short, any plane 